\title{
Qualitative Distances and Qualitative Description of Images for Indoor Scene Description and Recognition in Robotics
}

\author{
Zoe Falomir ${ }^{\mathrm{a}, \mathrm{b}}$, \\ ${ }^{a}$ Engineering and Computer Science Dep., \\ Universitat Jaume I de Castelló, Spain, and \\ ${ }^{\mathrm{b}}$ Mathematik und Informatik Fach., Universität \\ Bremen, Germany \\ E-mail:zfalomir@\{uji.es,gmail.com\}
}

\begin{abstract}
This thesis is focused on reducing the gap between the acquisition of low level information by robot sensors and the need of obtaining high level information for enhancing human-machine communication and for applying logical reasoning processes. To this end, approaches for qualitative and semantic image description and qualitative distance sensor interpretation were developed. Experimentation was carried out on different robotic platforms showing useful applications.
\end{abstract}

Keywords: Sensor data integration, Fuzzy set theory, Qualitative representation, Image understanding, Ontology, Description Logics, Similarity, Robotics

\section{Introduction}

The automatic extraction of knowledge from the world by a robotic system is still an unsolved task in Artificial Intelligence. A robotic agent is in contact with the world through its sensors which provide mainly numerical information. Sonar, infrared and laser sensors obtain distance information. Cameras obtain digital images that are represented internally as matrices of red, blue and green colour coordinates. All this numerical values obtained from the environment need a later interpretation to provide the knowledge required by the robotic agent in order to carry out a task. However, a real fact in human cognition is that people classify things as members of categories and attach linguistic labels to them. Moreover, the information that human beings can describe and remem- ber from what they see/feel/etc. is expressed using words, that is qualitatively.

As the information provided by the electronic components in a robotic agent is numerical, the approaches that first appeared in the literature giving an interpretation of this information followed a mathematical trend. In this thesis, this problem is addressed from the other side: its main aim is to process numerical data in order to obtain qualitative information as human beings can do.

\section{Contributions of the thesis}

The contents of this thesis [1] are overviewed and linked in Fig. 1.

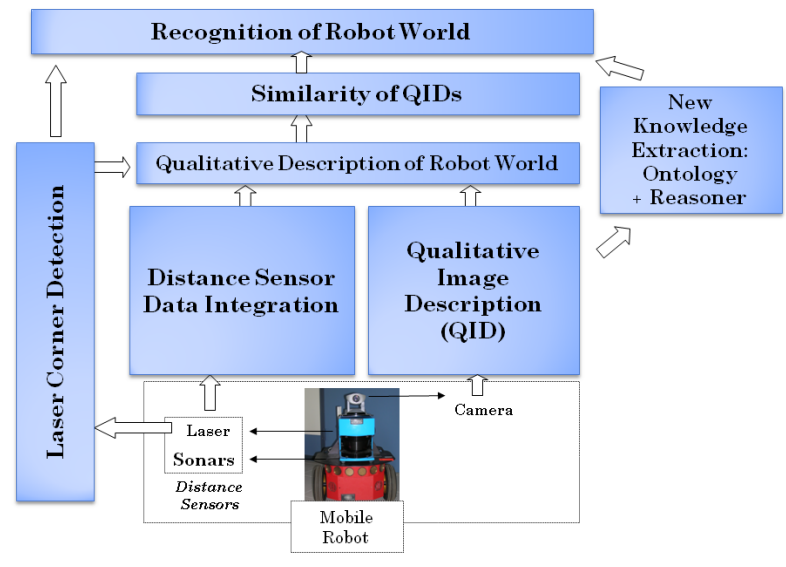

Fig. 1. Contributions of this $\mathrm{PhD}$ thesis.

The first contribution presented is a method for obtaining fuzzy distance patterns (built from qualitative distances with a degree of belief) obtained from the data provided by different distance sensors and the definition of a dissimilarity factor 
for comparing them. This method also: (i) integrates the distances obtained in order to detect special obstacles (glass windows, mirrors, etc); (ii) defuzzifies them to obtain a smooth robot speed, and (iii) applies them to classify pairs of corners (considered as indoor landmarks) into open reference systems (those defining spaces including more landmarks) or closed. To evaluate this method, an ActivMedia Pioneer $2 \mathrm{dx}$ mobile robot incorporating eight sonar sensors and a SICK LMS-200 laser range scanner has been used. The tests have been carried out in two real scenarios at a building of University Jaume I and the results show that this approach enables the robot to: (i) avoid crashing into obstacles when sensor disconnection happens, (ii) detect mirrors and glass windows as obstacles; (iii) approach obstacles at a smooth speed; and (iv) properly classify reference systems incorporating glass surfaces.

To introduce visual information in the description of indoor landmarks, a qualitative image description (QID) approach has been defined which describes digital images by extracting the visual and spatial features of their relevant regions/objects taking into account studies on how people describe images. New qualitative models for shape description and for colour description are defined for representing the visual features, while models of topology and qualitative orientation are used for describing the spatial features. In the validation of the QID approach, the results show that it is useful for describing images in two real robotworking scenarios: mosaic images captured by a camera AVT-Guppy F033C located on a platform from which a robot arm picks and places tile pieces to assemble mosaics automatically (Scenario I); and images of indoor corridors captured by a camera Logitech Quickcam Pro 9000 located on the top of a Pioneer 2 mobile robot (Scenario II). The QID approach has also proved to be independent of the image segmentation method used.

To provide a formal and explicit meaning to the QID generated, a Description Logic (DL) based ontology is built as the third contribution. Our approach automatically obtains a set of DL-axioms which describe a digital image visually and spatially. The objects included in the images are categorized according to the ontology schema and the DL reasoner into walls, floor, office doors and fire extinguishers. Tests carried out using digital images captured by a camera incorporated in a Pio- neer 2 mobile robot at the corridors of University Jaume I (Scenario II) showed that this approach can categorize objects under different illumination conditions and from different observer viewpoints.

In order to recognize QIDs (i.e., for identifying indoor landmarks), a similarity measure between QIDs is presented, which defines similarity measures between qualitative shape $(\operatorname{Sim} Q S D)$ and colour (SimQCD) descriptions, and between the spatial descriptions of topology and orientation.

The $\operatorname{Sim} Q S D$ defined is evaluated on images of different categories of the MPEG-7 CE-Shape1 library. The results obtained from the tests show that the SimQSD is invariant to rotations, translations, scaling and mirror-image changes of shapes and also combinations of these.

The $\operatorname{Sim} Q C D$ is combined with the $\operatorname{Sim} Q S D$ to define a pragmatic approach for assembling tile mosaics by approximate matching. The remarkable results of this application are that it accelerates previous mosaic assembling processes and it can produce perceptually visual different mosaics from a given design by just relaxing the colour similarity threshold.

For evaluating the SimQID, tests have been carried out in two scenarios that involve comparisons of: images of tile compositions (Scenario I), and images of landmarks (corners) detected by the laser sensor (Scenario II). From the results obtained in Scenario I, the SimQID proved its applicability in tasks that involve a human understanding image description, such as image classification and retrieval in databases in general, but specifically retrieval of vector-drawings, icon or clip-art image search by examples. After the evaluation of SimQID in Scenario II, the results showed that, by defining a precise similarity threshold, all the landmarks (corners) are correctly recognized. The SimQID calculus can be time consuming in the worst case. However, the SimQID calculus is computed by a separated thread, so that the robot can continue navigating while the recognition is in process.

\section{References}

[1] Z. Falomir. Qualitative Distances and Qualitative Description of Images for Indoor Scene Description and Recognition in Robotics. $\mathrm{PhD}$ thesis at Universitat Jaume I (Spain) and Universität Bremen (Germany), directed by Dr. M. Teresa Escrig and Prof. Christian Freksa, ISBN 978-84-695-3694-0, November 2011. http://www.tdx.cat/handle/10803/52897. 